\title{
Chemometrics as a Tool to Gain Insight into Fiber Rope Aging from Infrared Images
}

\author{
Ellen Nordgård-Hansen ${ }^{1}$, Håkon Jarle Hassel ${ }^{2}$, and Rune Schlanbusch ${ }^{3}$ \\ ${ }^{1,3}$ NORCE Norwegian Research Centre AS, Universitetsveien 19, NO-4630 Kristiansand S, Norway \\ enor@norceresearch.no \\ rusc@norceresearch.no \\ ${ }^{2}$ Idletechs AS, Pirsenteret, Havnegata 7, NO-7010 Trondheim, Norway \\ hakon.jarle.hassel@idletechs.com
}

\begin{abstract}
Fiber ropes are steadily gaining in popularity for offshore lifting purposes. One limiting factor is many fibers' low tolerance for high temperatures. Measurements of rope temperature and changes in thermo-physical properties are therefore highly relevant, a task which may be performed using an infrared camera. Chemometrics is one tool among the many techniques available for image processing. The present paper details results from applying chemometrics to infrared images obtained from recent cyclic-bend-oversheave testing. It is shown how this tool contributes to separating the various phenomena going on, like changes in thermal properties, vertical rope movement, surface degradation, and rope twist. A brief discussion on the applicability for real-life monitoring is also given.
\end{abstract}

\section{INTRODUCTION}

\subsection{Background}

Fiber ropes are steadily gaining in popularity for offshore lifting purposes. The reason is its high strength and lifetime, as well as lack of corrosion problems in water, combined with low weight. Consequently, replacing steel wire ropes with fiber ropes allows the same crane to lift more weight than before, since the weight of the rope itself is nearly eliminated. Alternatively, the same weight can be lifted by smaller cranes, possibly even on smaller ships.

Fiber ropes come in many varieties, with different properties, and thus each application requires careful selection of the right fiber rope type. This selection is a complex process, as stated by DNV GL (2015), «The ability of a synthetic fibre Ellen Nordgård-Hansen et al. This is an open-access article distributed under the terms of the Creative Commons Attribution 3.0 United States License, which permits unrestricted use, distribution, and reproduction in any medium, provided the original author and source are credited. line to carry load depends on the magnitudes and durations of tensions to be applied, the magnitudes and durations of preceding loading, and on the associated temperatures within the load-bearing material».

On this background, and since several of the partners in the SFI for Offshore Mechatronics (SFI for Offshore Mechatronics, 2019) are producing fiber rope cranes (MacGregor, 2017; National Oilwell Varco, 2018), monitoring techniques for fiber ropes was chosen as a topic for the SFI, starting in 2016. In the present article, we will focus on temperatures and thermal properties. Inspired by the work of Davies et al. (Davies et al., 2013), modelling and lab tests have already been performed by the group (Falconer, Nordgård-Hansen, \& Grasmo, 2018; Oland, Bossolini, Nielsen, Sørensen, \& Veje, 2017). Due to the importance of temperature in the degradation of fiber ropes, and due to the promising results so far, a thermal camera was subsequently used to observe fiber ropes during Cyclic Bend Over Sheave (CBOS) testing. This test method is particularly relevant for mimicking active heave compensation, which is important in offshore lifting.

\subsection{Chemometrics}

The sequence of images from a thermal video camera is a very high-dimensional time series. Interpreting such a megavariate stream of data can be overwhelming. The measurement noise in the data for each time point in each camera pixel may be random and complex to deal with in detail, since there is no pattern to white noise. However, the interesting variations in the data come from the underlying physical phenomena that cause systematic patterns of variation in the thermal video data (thermal friction effects, physical rope changes etc.). This may be picked up by a bilinear model. 
Principal Component Analysis (PCA) is a tool to extract systematic variation patterns from a data table, to gain knowledge about the most dominant patterns of covariation. It requires that the data is structured in rows representing the samples or observations, (e.g. time points), and columns representing variables or attributes (e.g. spatial positions, i.e. pixels). A data set $X$ with $n$ rows or observations and $m$ columns or pixels can then be described by the bilinear structure model of Eq. (1).

$$
X=T P^{\top}+E
$$

Where each column in $T$ is a time series score vector $t_{a} \in$ $\mathbb{R}^{n \times 1}$, and each column in $P$ is a spatial loading vector $p_{a} \in$ $\mathbb{R}^{m \times 1}, T=\left[t_{a}, a=1,2, \ldots, A\right]$ has in total $A$ columns, since the $A$ first PCs are intended to represent the main patterns of systematic variations among the $m$ variables. Conversely, the spatial loading matrix $P=\left[p_{a}, a=1,2, \ldots, A\right]$ represents the corresponding spatial covariation patterns. The last term, $E$, contains the unmodelled residuals. The first component describes as much as possible of the variance, the second one is orthogonal to the first and describes as much as possible of the remaining variance, etc., until all variance is described when $m$ components are included. If the optimal number of PCs, $A$, has been found, $E$ represents the remaining unsystematic errors, e.g. random measurement noise.

Mathematically, the columns of $P$ are the eigenvectors of the covariance matrix $X^{\top} X$. For images, each of these vectors can be seen as eigenimages, and represented as images. The scores tell how much of each of these images are required to represent each of the original images.

Most data of interest contain information that is replicated among its samples, and which has mutually dependent variables. This is where PCA is useful, through removing redundant information, whilst highlighting covariations. One might not be able to effectively discern different groups within the data by using univariate approaches but by looking at their covariances one could separate groups which otherwise would be difficult.

\subsection{Article structure}

The present article initially describes the experimental setup, including the software used for data analysis. Then, some modelling results are presented, followed by a discussion of how this leads to improved understanding of what happens to the rope during CBOS testing. Finally, implications for online real-life testing are discussed.

\section{EXPERIMENTAL}

\subsection{Equipment}

The ropes were tested on a machine for CBOS testing. The rope is mounted in a loop with a small test sheave at one end and a larger driving sheave at the opposite end. The sheaves then turn to make the rope move back and forth over the sheaves, resulting in wear from the repeated bending and straightening of the rope. A cylinder keeps the rope tension constant over time by increasing the sheave-to-sheave distance as required.

The machine is built by DEP Engineering in conjunction with NORCE, the University of Agder (UiA), and Mechatronics Innovation Lab (MIL), and is placed at the MIL's premises in Grimstad, Norway. Some technical details are given in Table 1.

Table 1. CBOS machine technical details

\begin{tabular}{|l|l|}
\hline Property & Value \\
\hline Rope Diameter [mm] & $20-30$ \\
\hline Machine Dimensions [m] & $12 \times 1.3 \times 2.2$ \\
\hline Test Sheave Diameter [mm] & $400-800$ \\
\hline Maximum Lune Pull [kN] & 150 \\
\hline Driving Sheave Diameter [mm] & 1000 \\
\hline
\end{tabular}

During cycling, a part of the rope never reaches the test sheave, while another part of the rope reaches the sheave and is just bent once before the sheave turns. This is rope in the so-called single-bend zone. Finally, a part of the rope reaches the sheave and is bent, but then goes on to be straightened out again after leaving the sheave at the bottom. This is rope in the so-called double-bend zone.

A Fluke 414D Distance Measuring Laser registers the cylinder position required to keep constant tension, thereby giving a measure of the rope creep.

Five optical cameras and one thermal camera registers the rope state during each test. In the present work, only the thermal camera data is used, which is of the type FLIR A6752sc. The image size is $640 \times 512$ pixels, and the accuracy is $\pm 1{ }^{\circ} \mathrm{C}$ or $\pm 1 \%$ of reading.

\subsection{Tests}

IR images were registered for tests on three different ropes, the first two of the same kind, the third of a slightly different kind, optimized for good bending performance. All ropes were 12-strand High-Modulus Polyethylene (HMPE) ropes having a diameter of $28 \mathrm{~mm}$.

The first two ropes were tested until they broke, after about 50000 cycles, or close to running a full week in time. The third rope did not break, but the test was stopped after about 70000 cycles, or just over a week, when there was severe rope damage close to the driving sheave.

\subsection{Data retrieval}

The thermal camera recorded images at $100 \mathrm{~Hz}$. In order to limit the data storage requirements, 2000 frames were recorded each 12000 second, meaning there is about 3.5 hours between each recording. 
For rope $1, \mathrm{csv}$ files were created from every $10^{\text {th }}$ frame from every recording, meaning there is about 200 files from each recording.

For rope 2, csv files were created from every $10^{\text {th }}$ frame from nine evenly spaced recordings from when the rope was new until it broke.

Fore rope 3, csv files were created from every $10^{\text {th }}$ frame from 35 evenly spaced recordings from the rope was new until the test was stopped.

\subsection{Software}

There are many different algorithms available for PCA. Most of them require that the data does not have any missing values. The bilinear On-The-Fly Processing (OTFP) modelling (Vitale et al., 2017) used here is a special method for dynamically developing a PCA model in cases where the data represents an "ever-lasting" stream of multi-dimensional input data that becomes too big to fit in the computer memory. The method was made available through Idletechs' OTFP software and accessed via Matlab scripts executed using Matlab R2018b.

\section{Modelling}

Different approaches were tested out, of which the following each gave useful insight:

- Limit in space: Static rope-only images

- Limit in content: Background removed

Since data from tests of three ropes was available, these could be modelled in different ways:

- One model can be used for all ropes

- $\quad$ Each rope can have its own model

Before modelling, the ambient temperature was subtracted, since the room was cold during start-up for rope 1 . The upper left pixel in each original image was used to estimate the ambient temperature.

First, models for rope 1 were built, and then this model was either applied to the remaining two ropes, or separate models were made for these.

\subsection{Limit in space}

The simplest way to reduce the amount of data to handle, is to select a static window which is always inside the rope, a Eulerian approach. An example is shown in Figure 1. A benefit of this approach is that vertical movement as well as loops and loose ends influence the pictures less than if the whole image was used. Since the rope's vertical movement is significant, the window is rather small.

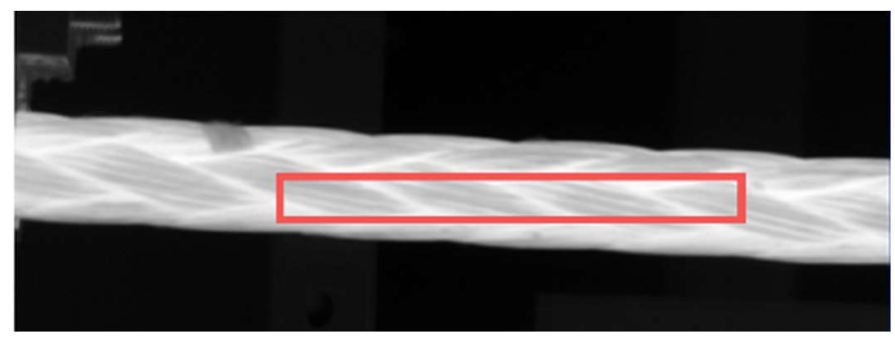

Figure 1. Example of limit in space

\subsection{Limit in content}

A simple form of dynamic background subtraction was applied to all images. In practice this meant that all pixels which had changed less than a threshold value on average during the previous 20 frames were given scaling 0 , while all other pixels were given scaling 1 .

\section{RESULTS}

\subsection{Limit in space}

\subsubsection{Rope 1}

Using this window for running PCA on all close to 7000 images files from rope 1 gave a first component which is clearly related to the average temperature variations within each cycle as shown in Figure 2 and Figure 3 and explained below.

In Figure 3, the scale goes from black for low values to white for high values. Even though some areas are colder than others, all pixels in the image shown in Figure 3 have positive values, meaning that any positive score indicates that all parts of the image has higher temperature than the center image shown in Figure 4, and any negative score indicates that all parts of the image has lower temperature than the center image. The net consequence is therefore a cyclic variation of the rope's average temperature.

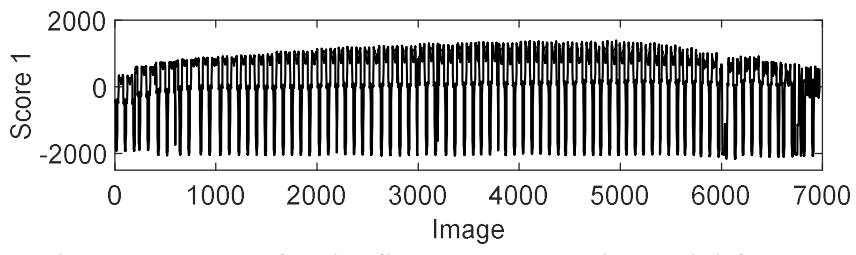

Figure 2. Scores for the first component in model from static rope-only window for rope 1

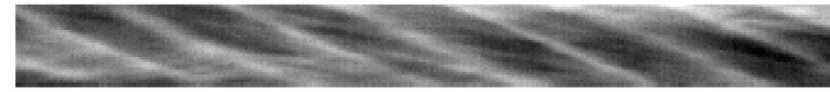

Figure 3. Loading for the first component from static ropeonly images for rope 1 
Figure 4. Center image from static rope-only images for rope 1

Zooming in, it is seen that the pattern changes from new rope, to spent rope, to close to broken rope, as seen in Figure 5.
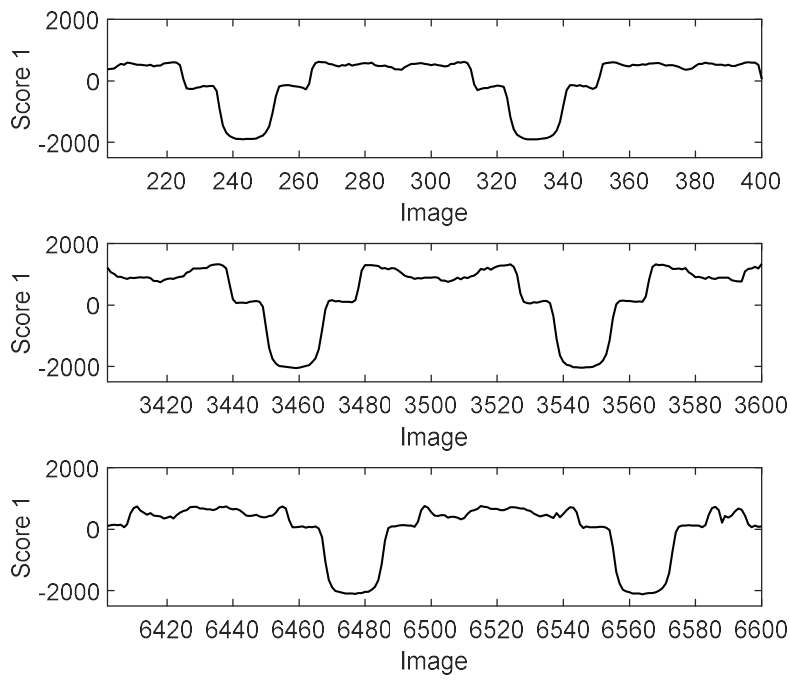

Figure 5. Score for the first component for new rope (top), spent rope (middle) and close to broken rope (bottom)

As seen from Figure 2 and Figure 5, the peak to shoulder ratio changes during the rope's lifetime. This is plotted in the upper part of Figure 6, where the high initial values are due to the low rope temperature relative to the ambient seen initially. Noteworthy is the clear decrease of this ratio observed when the rope nears the end of its lifetime. Similarly, the area between the shoulders of the score is plotted in the lower part of Figure 6. Towards the very end, the structure of the signal breaks down, but a clear area decrease is also observed earlier, related to the peak to shoulder ratio decrease.
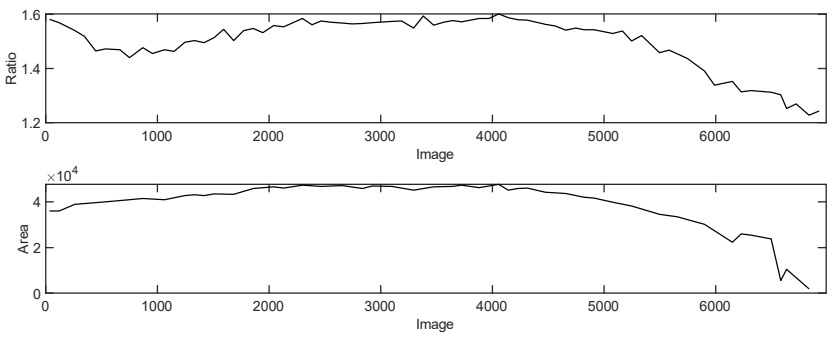

Figure 6. Peak to shoulder ratio and area between "shoulders" for score 1

The other components are only evaluated for the last few hundred images. Applying the final model to the whole set of images for rope 1 gives scores for all components. Most components show no very clear time development, or only some large values towards the very end of the rope's lifetime. Components 4 and 8 do show a clear time development, as seen in Figure 7 and Figure 8. The later components indicate temperature differences within each picture which are not clearly related to the rope's braiding structure, in clear contrast to the very structured loading for the first component, as shown in Figure 3.

The low importance of the components after the first one is also seen in the explained variance, which increased from $97.01 \%$ for one component to $97.08 \%$ for ten components.

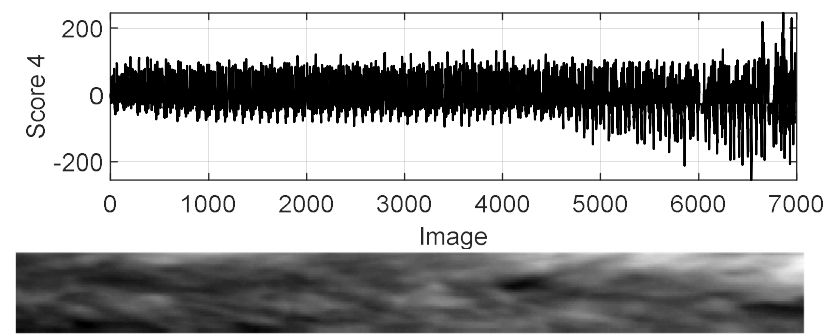

Figure 7. Scores and loading for component 4 from static rope-only window

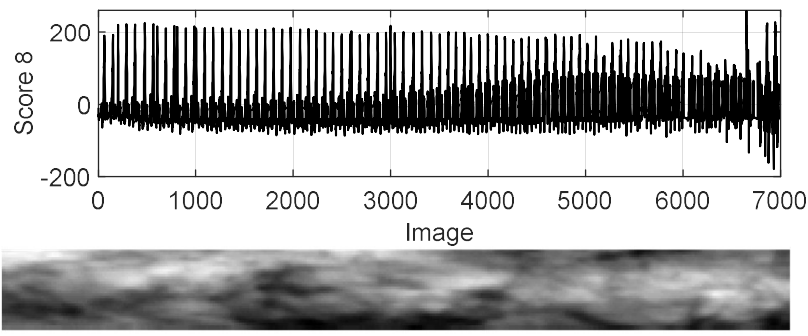

Figure 8. Scores and loading for component 8 from static rope-only window

\subsubsection{Rope 2}

Applying the model to the stored images from rope 2 gave score 1 as shown in Figure 9. One can see similar changes from initial flat tops of each cycle to "valleys" when rope gets older to a more chaotic shape towards the end of the rope's life time.

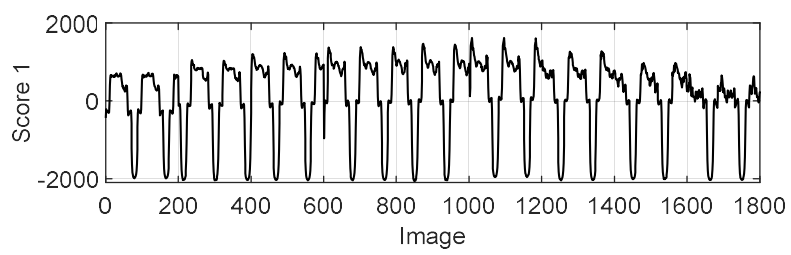

Figure 9. Score 1 for nine evenly distributed recordings for rope 2

Comparing the peak to shoulder ratio and the area between the shoulders for rope 2 gave similar trends as for rope 1 , even though the absolute values were somewhat different. The increased absolute values of the scores of components 4 and 8 are not prominent for rope 2 , but all scores show clear cyclic behavior also for rope 2 . 


\subsubsection{Rope 3}

Applying the model made from rope 1 on rope 3 gave values for score 1 as shown in Figure 10.

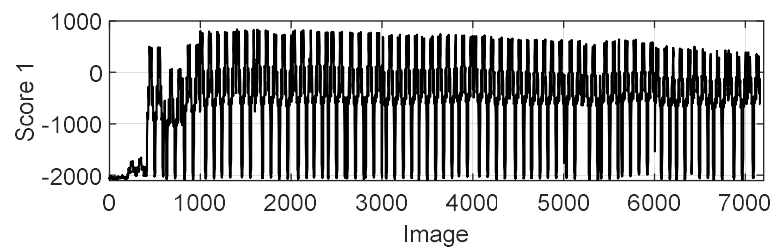

Figure 10. Score 1 from static rope-only images of rope 3, using the model from rope 1

Zooming in, it is seen that the scores change as shown in Figure 11. Though the shape is different from the shapes observed for ropes 1 and 2, similar changes over time are observed, with increased shoulder height initially, and later on lower reduced temperatures and breaking-up of the structure. The first recording (images $1-200$ in the upper part of the figure) was done before the test was started, and the second recording (images $200-400$ in the upper part of the figure) was recorded during start-up, before the rope had reached steady-state temperature.
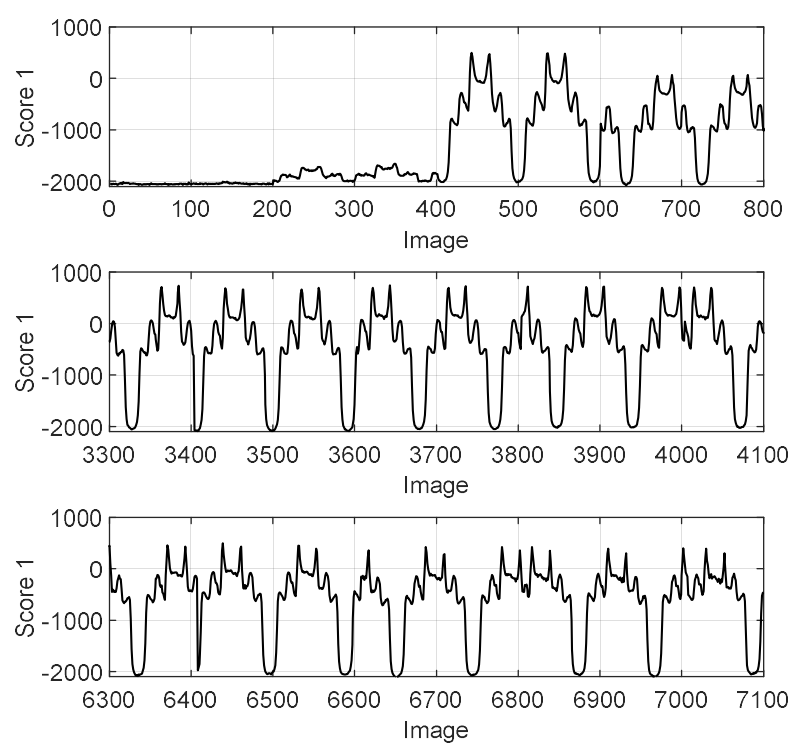

Figure 11. Score for the first component for new rope (top), spent rope (middle) and close to broken rope (bottom), rope 3 using the model from rope 1

\subsection{Limit in content}

\subsubsection{Rope 1}

The first component, as shown in Figure 12, mostly concerns the main temperature of the rope, visualized by the two rawimage examples in Figure 13. This is also seen by comparing with the scores from using the rope-only window in Figure 2.
Note the less-than-perfect symmetry of the scores from the current method.
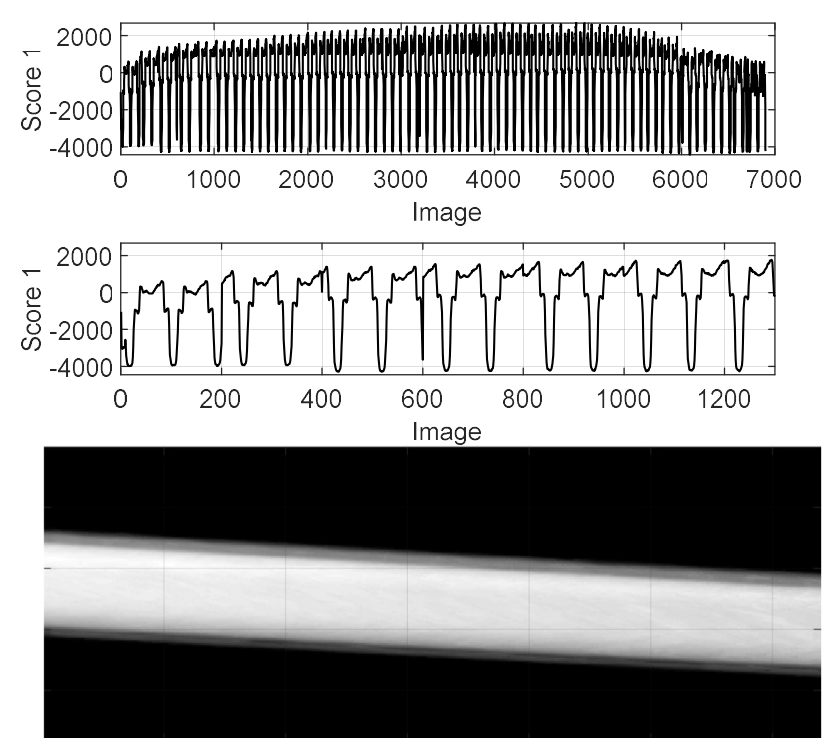

Figure 12. Scores and loading for component 1 from background-subtracted images
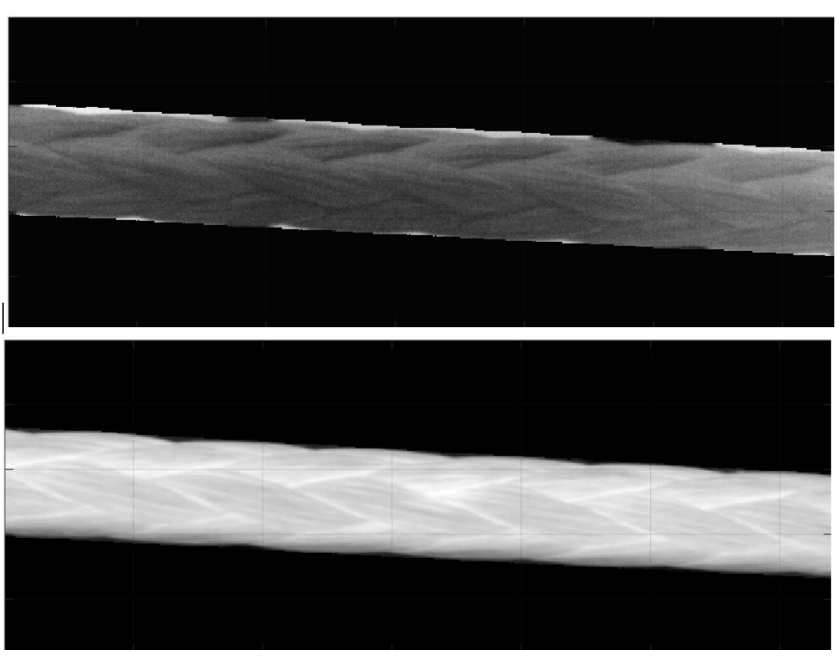

Figure 13. Raw images with low score (upper part) and high score (lower part) for component 1 from backgroundsubtracted images

The second component is related to a part of the CBOS machine appearing in front of the camera towards the end of the experiment, as seen in Figure 14. This is due to the machine stretching to keep constant tension in the rope while it elongates. As demonstrated by Falconer et al.(Falconer, Grasmo, \& Nordgård-Hansen, 2019), the rope elongation is significant, as well as being strongly localized. 

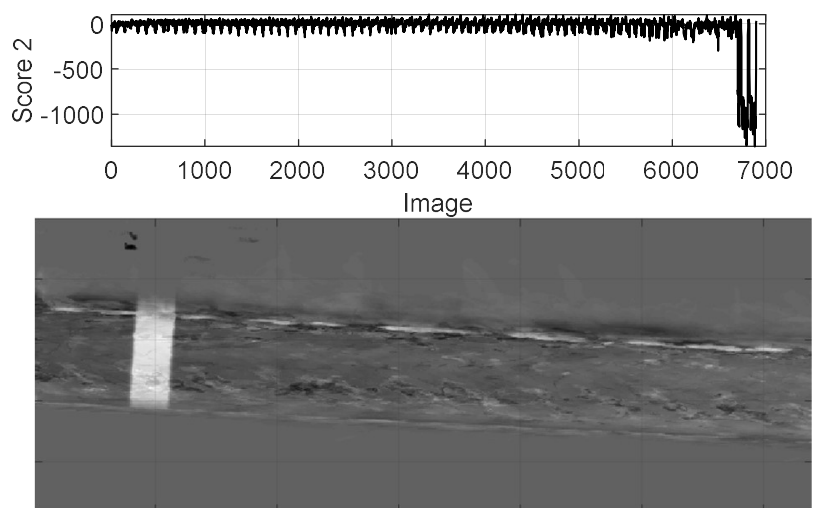

Figure 14. Scores and loading for component 2 from background-subtracted images for rope 1

The third component is related to vertical movement of the rope, as seen from Figure 15 and Figure 16.
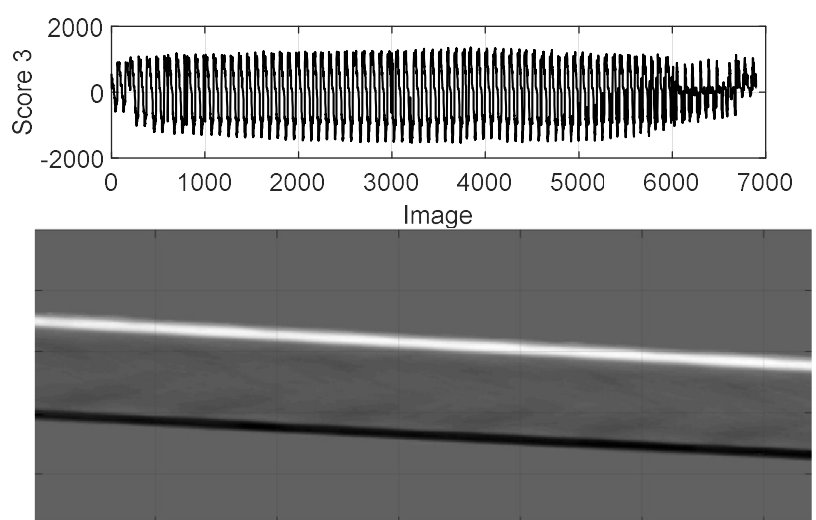

Figure 15. Scores and loading for component 3 from background-subtracted images for rope 1

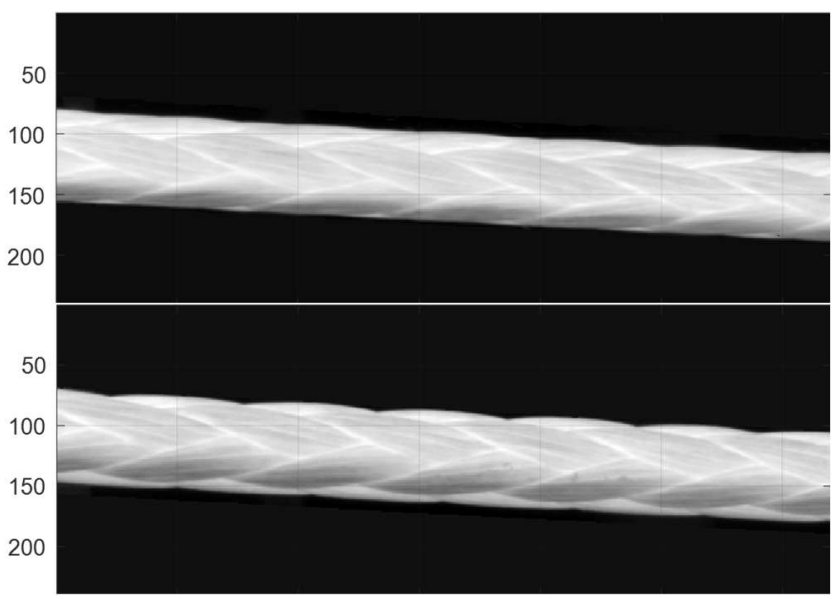

Figure 16. Raw images with low score (upper part) and high score (lower part) for component 3 from backgroundsubtracted images for rope 1
Components 4 to 7 all relate to rope surface changes towards the end of the rope's life time. Component 4 is shown as an example in Figure 15.

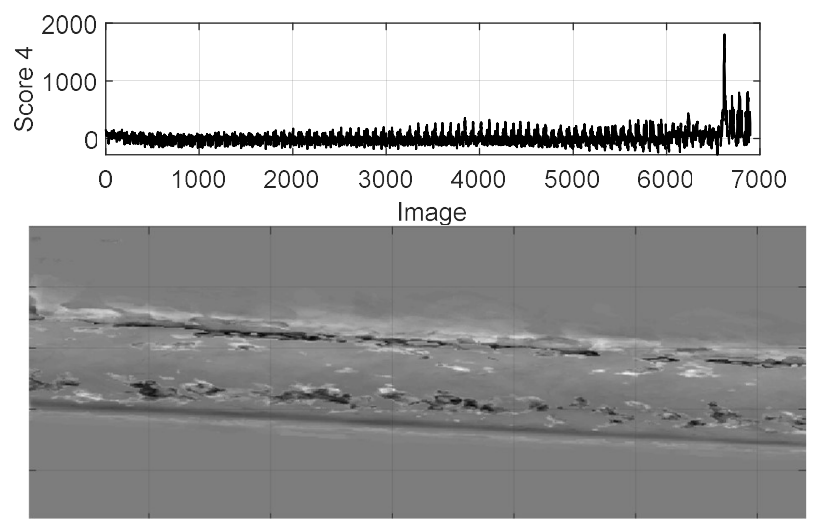

Figure 17. Scores and loading for component 4 from background-subtracted images for rope 1

The first, temperature-related, component explained $76 \%$ of the variation in the images, the second component another 12 $\%$, while including the vertical movement of the third component increased the total explained variance to $89 \%$. After 10 components, $91 \%$ of the total image variation was explained.

\subsubsection{Rope 2}

Applying the same method to make a specific model for rope 2 again gave temperature as the first component.

No part of the machine appeared in front of the camera in these tests. The second component was related to vertical movement, as seen in Figure 18.

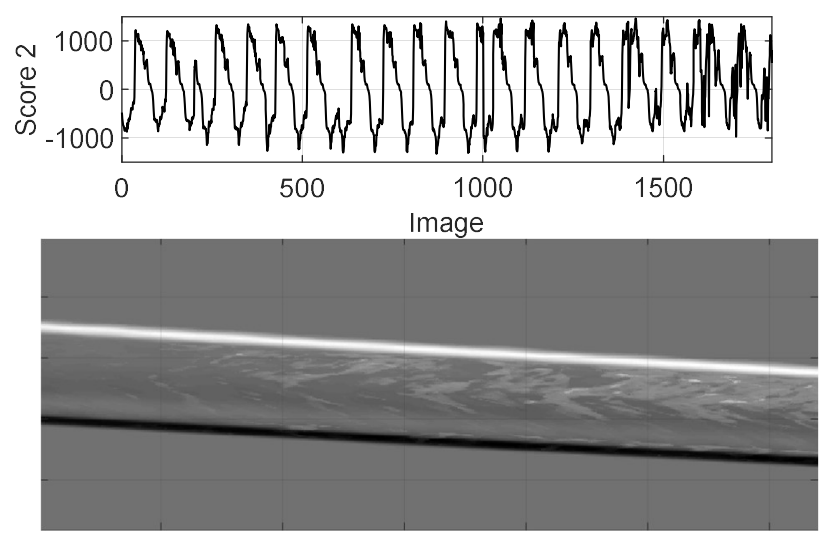

Figure 18. Scores and loading for component 2 from background-subtracted images for rope 2

The third and fourth components are related to surface changes as seen in Figure 19 for component 3. 

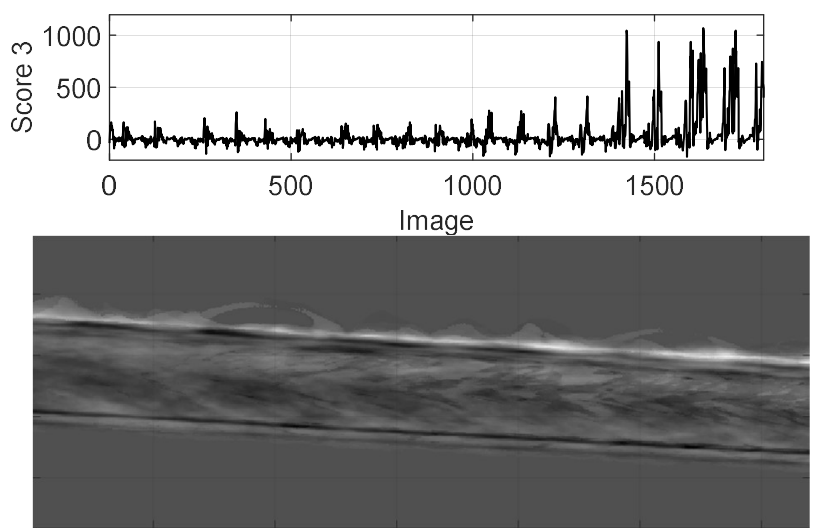

Figure 19. Scores and loading for component 3 from background-subtracted images for rope 2

The fifth component is related to vertical temperature distribution within the rope, as shown in Figure 20.

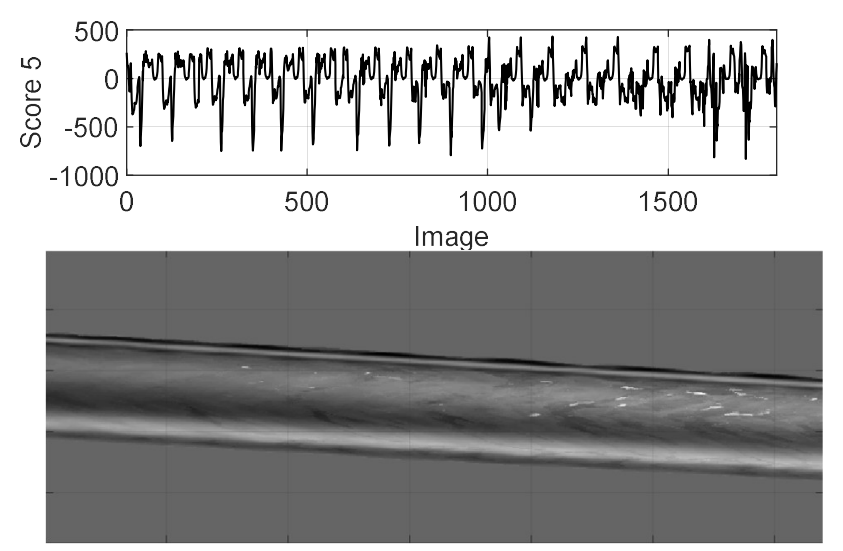

Figure 20. Scores and loading for component 5 from background-subtracted images for rope 2

Even though the corresponding explained variance is small, the scores for the fifth component showed a clear cyclic behavior, as illustrated in Figure 21.

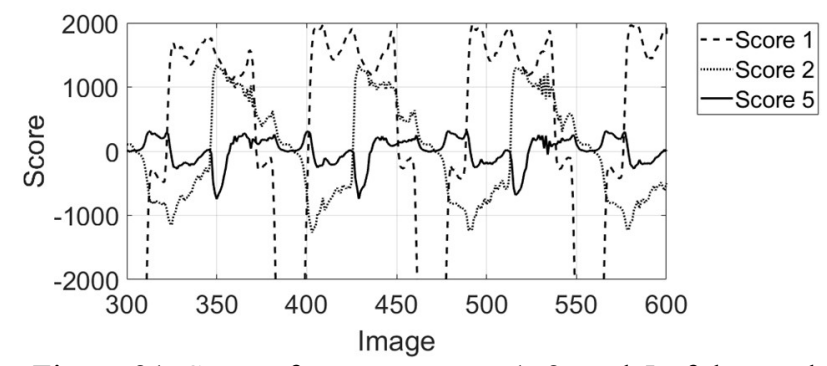

Figure 21. Scores for components 1, 2, and 5 of the model made from background-subtracted images from rope 2

When watching the corresponding videos, it is clear that for the single-bend zone, there is a cold zone below the middle of the rope, as seen in the upper part of Figure 22. When entering the double-bend zone, the temperature is more evenly distributed, though with low temperature at the very bottom, as shown in the middle part of the figure. Then, soon after the turn, the low temperature at the very bottom grows into a cold zone below the middle, as seen in the lower part of the figure.

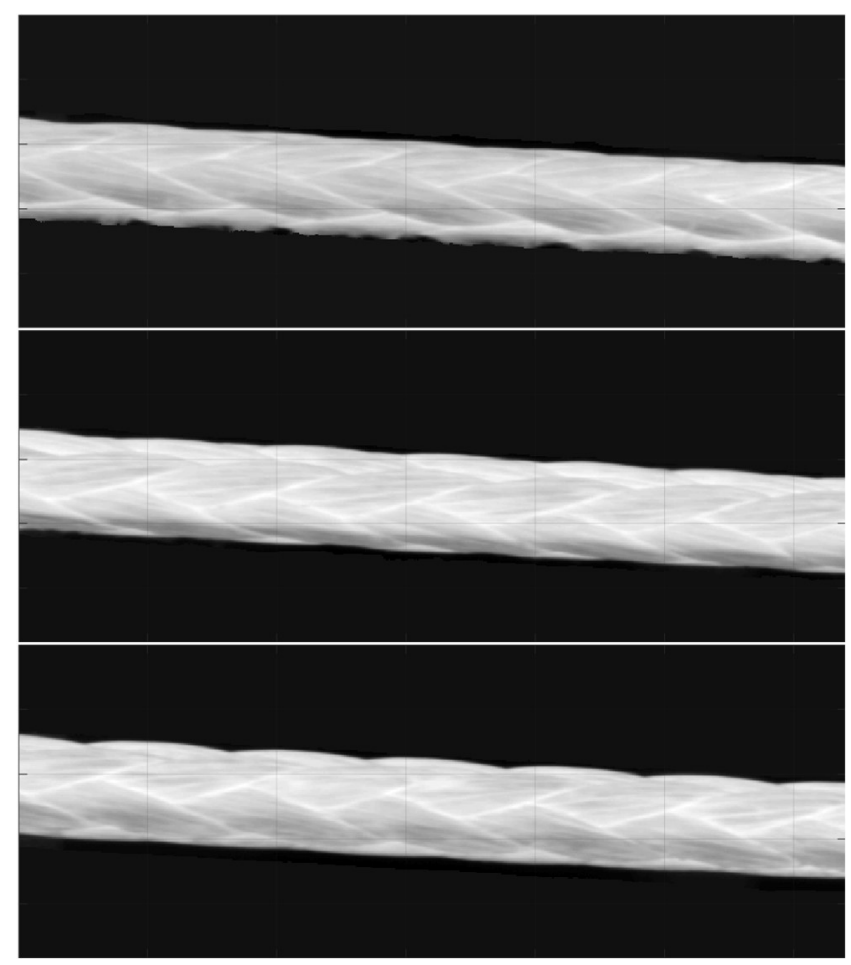

Figure 22. Vertical temperature variations - raw image examples near image 350

The first, temperature-related, component explained $76 \%$ of the variance, the second component, related to vertical movement, explained another $13 \%$, while about $2 \%$ was explained by the surface-related components 3 and 4 together. The fifth component explained less than $0.5 \%$ of the total image variations. Including all ten components explained in total $92 \%$ of the observed variance.

A model for rope 2 was also made without eliminating the background, though using the same vertical cropping. The results were similar, showing the same general trends in the scores, but there were less details in the loadings for the rope, and also some background present in the loadings.

\subsubsection{Rope 3}

For rope 3, the part from the CBOS machine was always in front of the camera, moving a bit towards the right from recording to recording. Still, the first component when making a fresh model for this rope was temperature-related, with scores very similar to for the space-limited model. The second component was related to the position of the CBOS machine part, and the third component to vertical rope 
movement. Components 4 and 5 contained more information on the machine part, while component 6 mostly contained twist, a cyclic property whose magnitude does not change much during the test. An example is given in Figure 23.

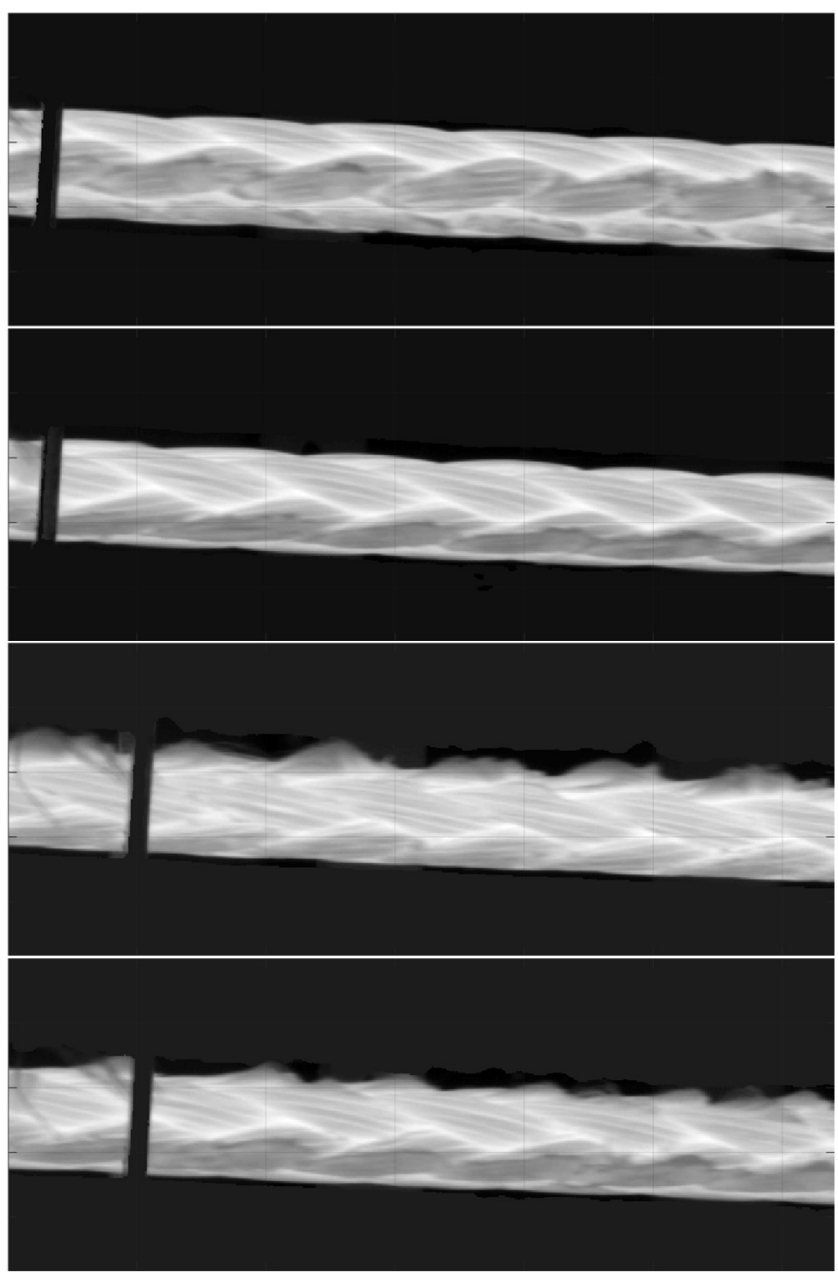

Figure 23. Component 6, low score new rope, high score new rope, low score spent rope, and high score spent rope

Apart from the machine part components, none of the scores for components 1-6 show a sharp increase in magnitude towards the end of test.

Neither of the first ten components are clearly related to surface changes, a fact that is also seen from watching the corresponding videos, where rope 3 exhibits far less surface degradation than the other two.

In total, all ten identified components explained just over 83 $\%$ of the observed image variation, of which the temperaturerelated first component explained $76 \%$. Another $5 \%$ was explained by the tool in the second component, and close to $0.5 \%$ was related to the vertical rope movement.

\subsubsection{Summary}

The allocation of the different components to physical phenomena is summarized in

Table 2, where each phenomenon is given a specific shading color.

Table 2. Component interpretations

\begin{tabular}{|c|l|l|l|}
\hline \multirow{2}{*}{ Comp. } & \multicolumn{3}{|c|}{ Rope } \\
\cline { 2 - 4 } & \multicolumn{1}{|c|}{$\mathbf{1}$} & \multicolumn{1}{c|}{$\mathbf{2}$} & \multicolumn{1}{c|}{} \\
\hline $\mathbf{1}$ & $\begin{array}{l}\text { Average } \\
\text { temperature }\end{array}$ & $\begin{array}{l}\text { Average } \\
\text { temperature }\end{array}$ & $\begin{array}{l}\text { Average } \\
\text { temperature }\end{array}$ \\
\hline $\mathbf{2}$ & CBOS part & $\begin{array}{l}\text { Vertical } \\
\text { movement }\end{array}$ & CBOS part \\
\hline $\mathbf{3}$ & $\begin{array}{l}\text { Vertical } \\
\text { movement }\end{array}$ & Surface & $\begin{array}{l}\text { Vertical } \\
\text { movement }\end{array}$ \\
\hline $\mathbf{4}$ & Surface & Surface & CBOS part \\
\hline $\mathbf{5}$ & Surface & Twist & CBOS part \\
\hline $\mathbf{6}$ & Surface & Surface & Twist \\
\hline
\end{tabular}

\section{DISCUSSION}

\subsection{Accuracy}

As demonstrated by the rope images shown earlier, the image size and temperature resolution are high enough to show rope temperature details. The images being clear and not blurred due to rope movement, further indicates that the image retrieval rate of $100 \mathrm{~Hz}$ was sufficient. The average rope temperature varies from around $20{ }^{\circ} \mathrm{C}$ to close to $60{ }^{\circ} \mathrm{C}$, meaning that the accuracy of $\pm 1{ }^{\circ} \mathrm{C}$ is not a problem here. Even the more subtle differences, e.g. related to surface degradation towards the end of rope 2's lifetime, is in the order of $10^{\circ} \mathrm{C}$.

The aim of the present study is to gain new qualitative understanding and to investigate the method for rope aging monitoring. On the other hand, detailed quantitative modelling of rope temperature changes is not the topic here. Therefore, relative temperature changes are of more interest than absolute changes. The properties of the thermal camera can therefore not be seen to impair the results discussed below.

\subsection{Improved understanding of cyclic bend over sheave}

The different models result in the same important factors describing what happened during the tests, as listed below:

- Average temperature

- Vertical temperature variations

- Surface changes

- Machine part in front of the camera

- Vertical rope movement

- Rope twist 


\subsubsection{Average temperature}

First, the average temperature is discussed, based on the findings from the space-limited model. A linear relation was found between the scores for component 1 and the average temperature deviation from ambient of these images. Therefore, one may conclude that $97 \%$ of the observed variations observed in this part of the images is explained by the average temperature alone.

The temperature cycles are explained in Figure 24 and Figure 25. In Figure 24, the sheave is seen on the right, and the green arrow represents the IR camera. An $x$ inside the sheave means that it is not moving, while arrows inside the sheave indicate rotation. The blue dots in Figure 24 represent the hot turning point, when the camera sees rope from the double-bend zone, which always has a bit of time to cool down (or to receive heat from the neighboring rope parts) between turns. This is marked with blue circles in Figure 25. The green dots and circles represent rope also from the double-bend zone, but which had no time to cool down after the previous turn. There are also the slightly different points, marked with light green circles in Figure 25. This the exact same rope position, but this time, the rope has had time to exchange heat with the ambient and with the rest of the rope before passing the camera. The orange dots in Figure 24 never pass the camera. The yellow circles in Figure 25 correspond to rope that has never been in contact with the sheave.

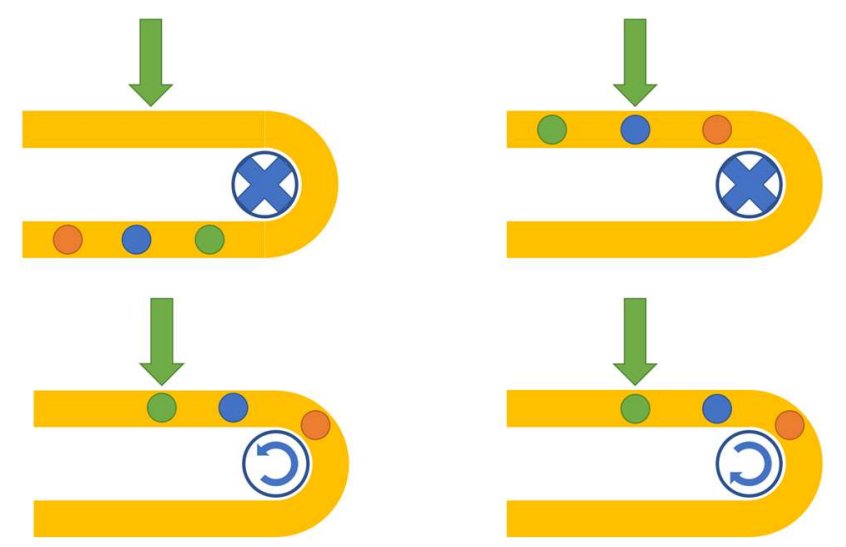

Figure 24. Temperature cycle explained

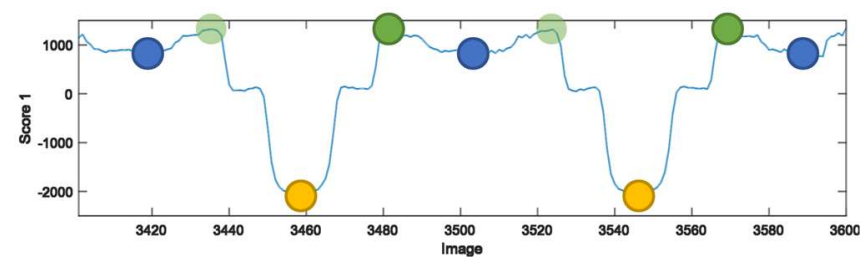

Figure 25. Temperature cycle explained as scores

The extreme symmetry observed between the peaks marked by dark and lighter green circles indicates that the heat exchange between the rope and the ambient by natural convection or by radiation is very limited for the rope portion observed by the static rope-only window. Otherwise, a cooling would be observed between the dark green and the light green circles. Alternatively, steady state may be reached, where the internal of the rope is cooled as heat escapes via the surface without the surface temperature changing.

The clear shape of each cycle in Figure 25 indicates that there is very little longitudinal heat conduction taking place within the rope. Otherwise, the heat from the double-bend zone would have «smeared» the very clear shoulders made by the singe-bend zone.

Different explanations for the observed temperature profile changes in time are discussed below.

Higher and sharper "horns" and shoulders may in principle have different reasons:

- Heat accumulation. This is refuted by the later collapse of the peaks.

- Increased heat transport to the surface. This could be caused by fusing of the strands, which reduces the heat transfer coefficients between the different strands.

- More heat generated at bending. This could be caused by stiffer strands as they age.

- Reduced heat capacity. As air moves out of the rope during this phase, the heat capacity actually increases, since the fibers' heat capacity is higher than that of air.

- Reduced density. This seems less probable, since the rope actually gets more compact during this stage.

Collapse of top towards the end may in principle also have various reasons:

- Reduced heat transport to the surface, which may be a result of many broken fibers, allowing more air into the rope, and introducing heat transfer coefficients between the strand ends.

- An increase in emissivity would mean that the registered temperatures are lower than the actual temperatures. In reality, the emissivity is expected to decrease as the surface gets rougher.

- Less heat generated at bending. This could be caused by more cut fibers or strands.

- Increased heat capacity. During this phase, strands are broken, allowing air into the rope. Since air has lower heat capacity than the fibers, the heat capacity will actually decrease.

- Increased density is less probable, since increased volume is observed during this stage.

To summarize, the observed changes are most likely caused by both changes in the heat transport to the rope surface and in the amount of heat generated upon bending.

Rope 3 shows a more complicated temperature profile for each cycle, as shown in Figure 26. Comparing to rope 1, it is 
seen that the difference is mostly in the top structure, but looking at the actual temperatures, it is found that the maximum temperature is not very different. The more complicated profile can be explained by even less longitudinal heat transfer than for ropes 1 and 2 . Rope 3 is said from the manufacturer to be optimized for good bending properties. One way to achieve this, is to coat each yarn or strand in order to reduce the inner friction. This would have the side effect of increasing the heat transfer coefficient between these rope parts, reducing the overall thermal conductivity.

Furthermore, the cycles for rope 3 are slightly longer, and a larger fraction of the rope passing the camera had been heated from bending. This is explained by rope 3 having a longer splice than the other ropes, forcing the test operator to shift the rope movement towards the driving sheave.

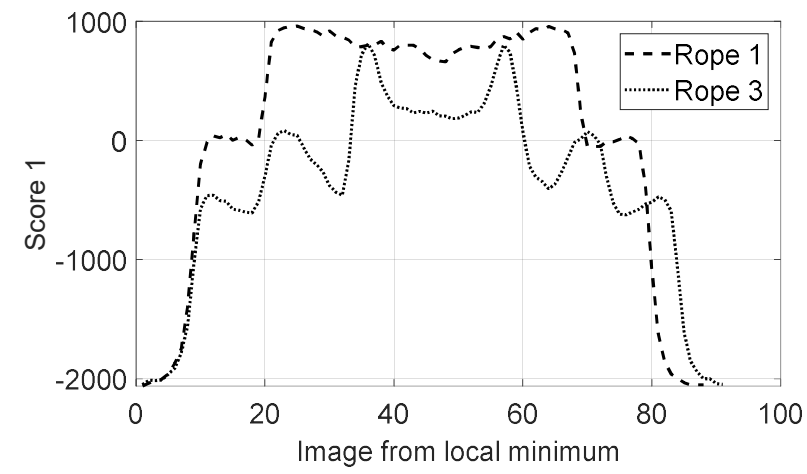

Figure 26. Score 1 for rope 1 and rope 3

\subsubsection{Vertical temperature variations}

The model made from background-eliminated versions of the entire images from rope 2 resulted in the fifth component being clearly related to variations in the vertical temperature profile. From this, it is observed that even though the average temperature of the double-bend zone does not change much when the sheave turns, the temperature distribution does. Watching the corresponding videos, it was observed that the root cause of this change is actually a twist of the rope, where the cold, lower part of the rope twists between the very bottom and just below the middle of the rope.

The lower part of the rope being colder in the first place may have several explanations:

- The bending mainly heats the rope near the top. This may be reasonable since there is more movement of the strands here than near the bottom, where the strands are compressed.

- The heat may be more readily conducted towards the top. Asymmetry in the rope's thermal properties may well be a result of asymmetry in heating of the rope.

- $\quad$ The rope may be cooled at the bottom from the sheave. Using a simple hand-held IR camera, it was observed that at least the sides of the sheave grooves were several degrees colder than the rope. It was not possible to measure the temperature at the groove bottom while an experiment was running.

\subsubsection{Surface changes}

When observing the rope tests with an untrained eye, the first feature that one sees, is the fraying of the rope's surface, and the occurrence of extruded loops after some time. This goes on for several days before the rope finally breaks. However, rope 2 did not look as bad as rope 1 when it broke, and it is hard to see which irregularities are only superficial and which are not.

These surface changes are clearly visible in the IR images. However, for the space-limited model, which only considered the central part of the surface, only $3 \%$ of the variation observed between all the $\approx 7000$ images from rope 1 were attributed to the combination of surface changes and other movement of the rope. On the other hand, surface changes resulted in significant components when the rope edges were also taken into account. The models made from the entire pictures for ropes 1 and 2 both gave scores related to surface changes which clearly increased as the rope got older. For rope 3 , this was not the case, possibly because the rope was not tested until it broke, but it was also noted that the surface changes that did occur, were far less random than for ropes 1 and 2 .

\subsubsection{Machine part in front of the camera}

This happened during the final recording of rope 1, while the part was present during the entire test of rope 3 . For the ropeonly window, the window was chosen such that the machine part was never in it.

For the model made from the entire images, with background subtracted, separate components were devoted to this feature. For rope 1, the part was only visible during the last recording, meaning it had only one position. Even so, component 2, which has this as its most prominent feature, explained $12 \%$ of the variation in the $\approx 7000$ images. For rope 3 , three components were mostly devoted to this machine part. Thus, chemometrics separates this feature from other phenomena, but it may still lead to other, more rope-related features getting less weight and attention than they would otherwise.

First, one should seek to avoid the problem in the physical machine-camera setup. If not possible, another improvement may be to crop the images so that the machine part is never visible, or to modify the background elimination to also eliminate this part.

\subsubsection{Vertical rope movement}

Vertical rope movement is observed in the models made from the entire images with background eliminated. Figure 27 shows the scores of components 1 and 3 plotted together for 
the model made for rope 1 . Note that the vertical movement follows the machine's cycles very systematically.

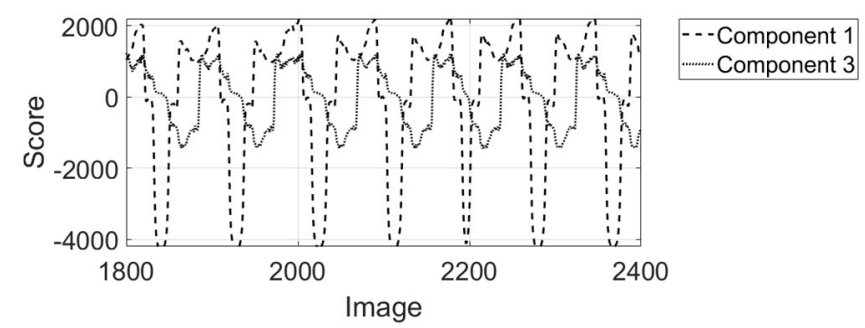

Figure 27. Scores from components 1 and 3 plotted together

The sharp increase observed near the hot turning-point, i.e. when rope from the double-bend zone is next to the camera and the sheaves do not move, might indicate that the rope tension is always updated at this point of the cycle. However, looking at the cylinder position as a function of time in Figure 28 , it is seen that the tension is not increased that regularly.

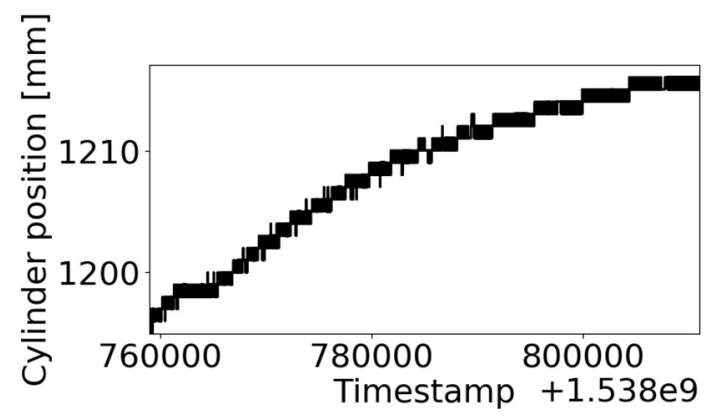

Figure 28. Cylinder position as a function of time

\subsubsection{Rope twist}

In the model made from images without background for rope 3 , rope twist was an important part of the sixth component. The score for this component varied cyclically, indicating that the twist was a feature of how the rope was mounted, and not a feature of the rope aging.

For ropes 1 and 2, either there was less twist present in the rope, or the twist was overshadowed by the more severe surface degradation observed there.

\subsection{Rope differences}

Even though the first component describes average temperature variations in all cases, different physical phenomena are related to different components for the different ropes. This is summarized in Table 2 for analysis limited in content.

Since the CBOS part is never in front of the camera for rope 2 , it is natural that this phenomenon is not related to any changes observed in the images for this rope. The CBOS part is in all images for rope 3, but only in the images from the final recording for rope 1 . Therefore, it is also natural that more than one component is required to describe the image changes related to the CBOS part for rope 3 .

For rope 3, the twist was present during the whole process, as shown in Figure 23. Analyzing the vertical temperature differences of component 5 for rope 2, it was seen that this was also related to rope twist, present during the whole process.

As discussed earlier, rope 3 was not run until it broke at the testing sheave, so it did not exhibit severe surface damage. This is the reason for no surface damage related components in the analysis of the images from rope 3.

It can therefore be concluded that the present tests indicate that the physical phenomena observed can be ordered according to how important they are for the IR images as follows:

1. Average temperature

2. CBOS part in front of camera, if present

3. Vertical rope movement

4. Surface degradation, if present

5. Rope twist, if present

Doing experiments on different ropes over time is likely to result in different phenomena influencing the images to various degrees, such as machine parts in front of the camera, various degrees of twisting, etc. An important benefit of the present method is that it separates the phenomena, so they can either be analyzed separately, or some phenomena may be disregarded.

\subsection{Modelling approaches}

In addition to the methods described in detail in the present paper, other approaches were also tried out. These are listed below:

- Limiting in time, i.e. looking at changes over time for one specific part of the rope - a Lagrangian approach.

- Selecting similar images using PCA to define important features that describe similar images, and then making a model using these images only.

- Using warp and shift to generate modified images where the rope seems to always be in the same place, and then making a model from these.

- Stacking all images from one cycle per recording to make large hyper-images, and then making a model from these.

Using the first of these approaches on rope from the doublebend zone, it was observed that different tests on the same type of rope may give highly different quantitative measures of surface changes in the double-bend zone. A reason may be that details in e.g. rope mounting and wear of the sheave influences how the surface develops, making one unified 
model infeasible. When making one model for each rope, both models resulted in score values that increased sharply when the rope was nearing the end of its lifetime. Using the same method, the second component for rope 1 was related to localized rope twist. This was not found for this rope for the model considering larger portions of the rope. On the other hand, this method did not detect vertical movement, since it only considered a very small part of each cycle.

The second approach inspired the first approach, since PCA on some manually selected very similar images gave temperature as the most important component. Images with similar temperatures are from the same part of the cycle, and thus from the same physical part of the rope. Up-weighing less significant components led to considering vertical movement or surface degradation, again strongly linked to cycle position. For less regular, and more realistic, tests, these two methods will naturally be more diverse, and PCA may be more useful than simply picking images from the same part of the cycle.

The third approach required frequent reference images, and even then, the image processing was only partially successful. Therefore, the results showing how to build up these warped imaged were hard to interpret.

Finally, the fourth method revealed the vertical rope movement very clearly and showed temperature changes within the single- and double-bend zones as well as vertical temperature differences. However, there was some confounding between different phenomena, where one component could e.g. be related to both vertical movement and to fraying.

To conclude, the best approach depends on the goal of the investigation, since different approaches can detect different features of the test or the rope.

\subsection{Fiber rope monitoring}

The ropes all show clear, cyclic temperatures profiles, which change in a characteristic way as the rope ages. These are readily available from IR images by simply finding the average temperature in a window which is placed to be always within the rope. Physically, the time development is explained as changes in the rope's thermal conductivity and in the heat generated by bending.

For use in real-life monitoring of fiber ropes, one must consider how these profiles will look when different sections of the rope are subject to bending at varying time intervals. The underlying physical changes will be the same, so clear changes are expected. However, the temperature profiles will not be as clear as from the very regular testing performed here.

Chemometrics shows promise in separating various phenomena, such as temperature change, vertical rope movement, surface degradation, etc. This is in line with the findings in (Vitale et al., 2017), where chemometrics was also used to improve the understanding of the experiments by discovering and extracting systematic covariance information from data streams.

When using IR images to monitor surface degradation, it is seen that separate models must be made for each specific rope being tested. Doing so, scores for the surface-related components exhibit clear increases as the rope ages. A problem is that since each model is made for a specific rope, it is hard to quantify the remaining useful life based on current score values. Rather, one most look at how the score values change over time or look for emerging components with loadings indicating very large changes.

When the method is generalized as described above, scores from relevant components may be combined to give one indicator of rope age.

Finally, the modelling compresses the data, by removing redundancy and decomposing the statistically significant variation into principal components. This reduces the number of components to keep track of, making it feasible to do online monitoring of the knowledge gained. For each component, one image and a vector the length of the desired time window is necessary. Additionally, a center and scaling image is needed. As a simplification, neglecting the need for model updates, the storage requirement using an established model, relative to the original data, is number of components / number of variables. In the case of a $640 \times 512$ pixel image with 10 components, this is as given in Eq. (2).

$$
\frac{10}{640 \cdot 512}=\frac{10}{327680}=\frac{1}{32768}=3.1 \cdot 10^{-5} .
$$

\section{FURTHER WORK}

In order to learn more about what causes the observed changes, thermocouple measurements within the rope during CBOS testing would be useful.

To move towards real-life offshore testing, changes in the rope's average temperature with more irregular bending, both in position and in time, should be considered and tested.

Also, for real-life testing, remaining useful life must be quantified from the observed changes.

\section{CONCLUSION}

A fiber rope's thermal properties do change during its lifetime, and these changes are detectable using an IR camera.

Chemometrics shows promise in separating changes in average temperature from surface changes, rope movement, etc. Therefore, when analyzing IR images from rope testing in order to improve the process understanding, chemometrics is a useful tool. 
If IR images are used to monitor fiber rope surface deterioration for maintenance purposes, chemometrics can be useful for setting up suitable models.

\section{ACKNOWLEDGEMENT}

The authors would like to thank Shaun Falconer for running the experiments, Benyamin Akdemir for valuable discussions, and the University of Agder for access to the IR camera.

The research presented in this paper has received funding from the Norwegian Research Council, SFI Offshore Mechatronics, project number 237896.

\section{REFERENCES}

Davies, P., Lacotte, N., Kibsgaard, G., Craig, R., Cannell, D., François, S., Lodeho, O., Konate, K., Mills, S., François, M., Defoy, A.-L., Durville, D., Vu, D., Gilmore, J., \& Sherman, D. (2013). Bend over sheave durability of fibre ropes for deep sea handling operations. ASME 2013 32nd International Conference on Ocean, Offshore and Arctic Engineering, Nantes, France.

DNV-GL. (2015). Offshore standard DNVGL-os-e303 offshore fibre ropes: DNV GL

Falconer, S., Grasmo, G., \& Nordgård-Hansen, E. (2019). Condition monitoring of HMPE fibre rope using computer vision during $\mathrm{CBOS}$ testing. OIPEEC Conference, The Hague, Netherlands.

Falconer, S., Nordgård-Hansen, E., \& Grasmo, G. (2018). Temperature measurements as a method for monitoring ropes. WCEAM 2018: The 13th World Congress on Engineering Asset Management, Stavanger, Norway.

MacGregor (Producer). (2017). Products \& solutions: Fibre rope crane. Retrieved from https://www.macgregor.com/Productssolutions/products/offshore-and-subsea-loadhandling/fibre-rope-crane/

National Oilwell Varco (Producer). (2018). Next generation subsea knuckle boom crane. Retrieved from https://www.nov.com/Segments/Rig_Technologies/Mar ine and Construction/Lifting and_Handling/Product Catalogue/Cranes/Next_Generation_Subsea_Knuckle_ Boom Crane.aspx

Oland, E., Bossolini, E., Nielsen, O. W., Sørensen, M. P., \& Veje, C. T. (2017). Modelling the thermal properties of large diameter fibre ropes. OIPEEC, La Rochelle, France.

SFI for Offshore Mechatronics. (2019). from https://sfi.mechatronics.no/

Vitale, R., Zhyrova, A., Fortuna, J. F., de Noord, O. E., Ferrer, A., \& Martens, H. (2017). On-the-fly processing of continuous high-dimensional data streams. Chemometrics and Intelligent Laboratory Systems(161), 118-129.

\section{BIOGRAPHIES}

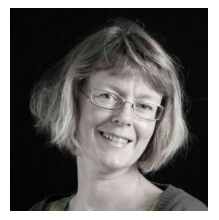

Ellen Nordgård-Hansen received her MSc in Physical Chemistry from NTH in Trondheim in 1995, and a PhD in NonEquilibrium Thermodynamics in 1997. In the following years, she worked with modelling and control at Prediktor in Fredikstad, Norway, before she joined Projectiondesign, also in Fredrikstad, to work on designing projector cooling. Since 2015, she holds position as Senior Researcher at Teknova, now NORCE Norwegian Research Centre. Her research interests include modelling and simulation, both data based and from first principles, programming, regulation and control.

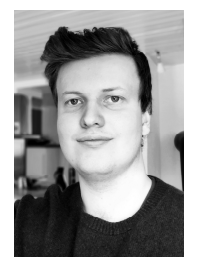

Håkon Jarle Hassel received his M.Sc. in the field of artificial intelligence from the Norwegian University of Science and Technology (NTNU) in Trondheim, Norway 2017. He previously received his B.Sc. in computer science from Sør-Trøndelag University College (HiST) in Trondheim, Norway 2015. He started working for Idletechs in 2017, where he provides analysis support to customers and assists in running experiments and software development. He particularly enjoys working together with domain experts to uncover physical variation patterns contained in datasets.

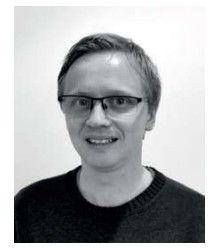

Rune Schlanbusch received his MSc in Space Technology from Narvik University College (NUC), Narvik, Norway in 2007, and $\mathrm{ahD}$ in Engineering Cybernetics from NTNU, Trondheim, Norway in 2012. He currently holds positions as Senior Researcher at The Norwegian Research Center (NORCE) and CTO at the startup company Machine Prognostics, which is a spin-off based on research within condition monitoring technologies. His major research interests include nonlinear control theory and stability analysis, Multiphysics modeling and simulation, intelligent sensing technologies, condition monitoring, and drone technology. He is currently a member of the IEEE since 2008 within the control, robotics and communication societies. 